\title{
Sensibilité des bilans de masse glaciaires aux fluctuations climatiques
}

\author{
M. Vallon, C. Vincent et L. Reynaud \\ Laboratoire de Glaciologie et Géophysique de l'Environnement du C.N.R.S. et Université Joseph Fourier
}

Il y a une dizaine d'années, Mark Meier (1984) suggéra que les «petits" glaciers pouvaient porter une lourde responsabilité dans l'élévation du niveau des mers au cours du $\mathrm{XX}^{e}$ siècle. Depuis, l'estimation de la sensibilité du bilan de masse de ces «petits» glaciers aux fluctuations de température a été largement révisée à la baisse [1, 2, 3], leur contribution passant, entre 1984 et 1992, de 469 à $209 \mathrm{~km}^{3}$ d'eau par an et par degré [4]. Si les «petits" glaciers d'Alaska et de Patagonie semblent jouer un rôle de plus en plus modeste dans les fluctuations du niveau des mers, les glaciers des Alpes contribuent en revanche pour une large part au remplissage des retenues hydroélectriques. L'analyse de leur sensibilité aux fluctuations climatiques est d'autant plus intéressante que nous pouvons confronter pour quelques glaciers des Alpes françaises l'estimation des bilans de masse aux mesures de terrain.

Pour ce faire, deux méthodes peuvent être envisagées : on peut, à la suite de Martin [5], chercher une relation empirique entre variabilité climatique et fluctuations des bilans de masse ou, comme Oerlemans [6] par exemple, tenter d'estimer les variations des bilans de masse glaciaires à partir de l'analyse de l'évolution probable des différents termes du bilan énergétique. Les deux méthodes semblent conduire à des ordres de grandeur comparables : la sensibilité des glaciers de montagne serait, du moins pour ce qui concerne les glaciers des Alpes et de Scandinavie, les mieux étudiés à ce jour, d'environ $-0,4$ à $-0,6 \mathrm{~m}$ de glace. ${ }^{\circ} \mathrm{C}^{-1}[7,8,9,6]$. Cette sensibilité est assez nettement différente de celle que l'on peut attendre avec le modèle de fluctuations interannuelles des bilans de masse indépendantes de l'altitude proposé par Lliboutry en 1974 [10]. Dans ce cas, avec un gradient de température d'environ $-7^{\circ} \mathrm{C} \cdot \mathrm{km}^{-1}$ et un gradient de bilan en zone d'ablation de +5 à $+7 \mathrm{~m}$ d'eau. $\mathrm{km}^{-1}$ on devrait observer une sensibilité de $-0,7$ à $-1,0 \mathrm{~m}$ d'eau. ${ }^{\circ} \mathrm{C}^{-1}$.

Ce coefficient de sensibilité, qui conditionne tant la variation annuelle du volume des glaces que le volume final de celles-ci à la suite d'une modification climatique, mérite un examen plus attentif. La notion de relation caractéristique entre bilan spécifique et variation climatique de la forme $b_{s}=(\alpha \times \mathrm{d} T)+(\beta \times \mathrm{d} P)$, où $b_{s}$ est le bilan spécifique du glacier, $\mathrm{d} T$ une variation de température, $\mathrm{d} P$ une variation de précipitation à la station météorologique de référence, $\alpha$ et $\beta$ des coefficients à déterminer, ne peut en effet se concevoir que dans le cadre du modèle linéaire de Lliboutry (op. cité). Or, un tel modèle n'est valable qu'à l'échelle où il a été établi, celle d'un glacier de quelques $\mathrm{km}^{2}$ et Kuhn [11] a suggéré qu'une approche non linéaire serait plus réaliste sous d'autres climats. Mais, même dans les Alpes, il est peu raisonnable de prétendre qu'une variation de la température estivale de $1{ }^{\circ} \mathrm{C}$ ou une variation des précipitations hivernales de $10 \mathrm{~cm}$ d'eau à Chamonix se traduira par la même variation de bilan de masse à $1300 \mathrm{~m}$ d'altitude, au front du glacier des Bossons et à $4800 \mathrm{~m}$, au sommet du Mont Blanc.

La réponse des bilans de masse glaciaires aux modifications climatiques ne peut être, a priori, que très fortement dépendante de l'altitude : à haute altitude, dans les régions où les échanges turbulents jouent un rôle négligeable, les variations de température de l'air auront beaucoup moins d'importance qu'à basse altitude. Inversement, dans les massifs montagneux, compte tenu de l'augmentation des précipitations avec l'altitude, les fluctuations de précipitation seront probablement d'autant plus efficaces que l'altitude est élevée. Le concept de sensibilité du bilan spécifique aux fluctuations climatiques nous semble donc devoir être remplacé par celui de sensibilité de la relation bilan de masse-altitude à ces fluctuations.

\section{I $\square$ SENSIBILITÉ DE LA RELATION BILAN DE MASSE-ALTITUDE AUX VARIA- TIONS CLIMATIQUES}

Pour tenter de préciser cette relation, nous disposons de la série d'observations réalisées sur le glacier d'Argentière par le Laboratoire de Glaciologie et Géophysique de l'Environnement du C.N.R.S. depuis 1975. Sur ce glacier, les bilans de masse sont observés à quatre altitudes différentes entre 1850 et $2700 \mathrm{~m}$ (fig. I) et la station météorologique de Chamonix $(1000 \mathrm{~m})$ se trouve à quelques kilomètres du glacier. La période d'observation peut paraître courte (18 ans) mais, étudiant la stabilité de la relation bilan spécifique-climat pour le glacier de Sarennes, C. Gelbert [12] a montré que, si des périodes d'apprentissage de 5 à 10 ans étaient tout à fait insuffisantes pour établir une relation fiable, dès que l'analyse statistique portait sur une période supérieure à 15 ans, les variables explicatives ne changeaient pas et les coefficients de régression demeuraient stables.

Chacun des sites d'observation (vers 1850, 2400, 2550 et $2700 \mathrm{~m}$ d'altitude) est une zone d'environ 25 hectares où, suivant les années, les bilans de masse ont été mesurés sur 3 à 10 balises. La variabilité spatiale des bilans de masse, liée à l'exposition, la pente, la couverture morainique ou nivale, étant particulièrement importante sur les profils situés à 1850 et 2700 mètres (gradients de l'ordre de 1 à 2 mètres de glace par an et par hectomètre tant 


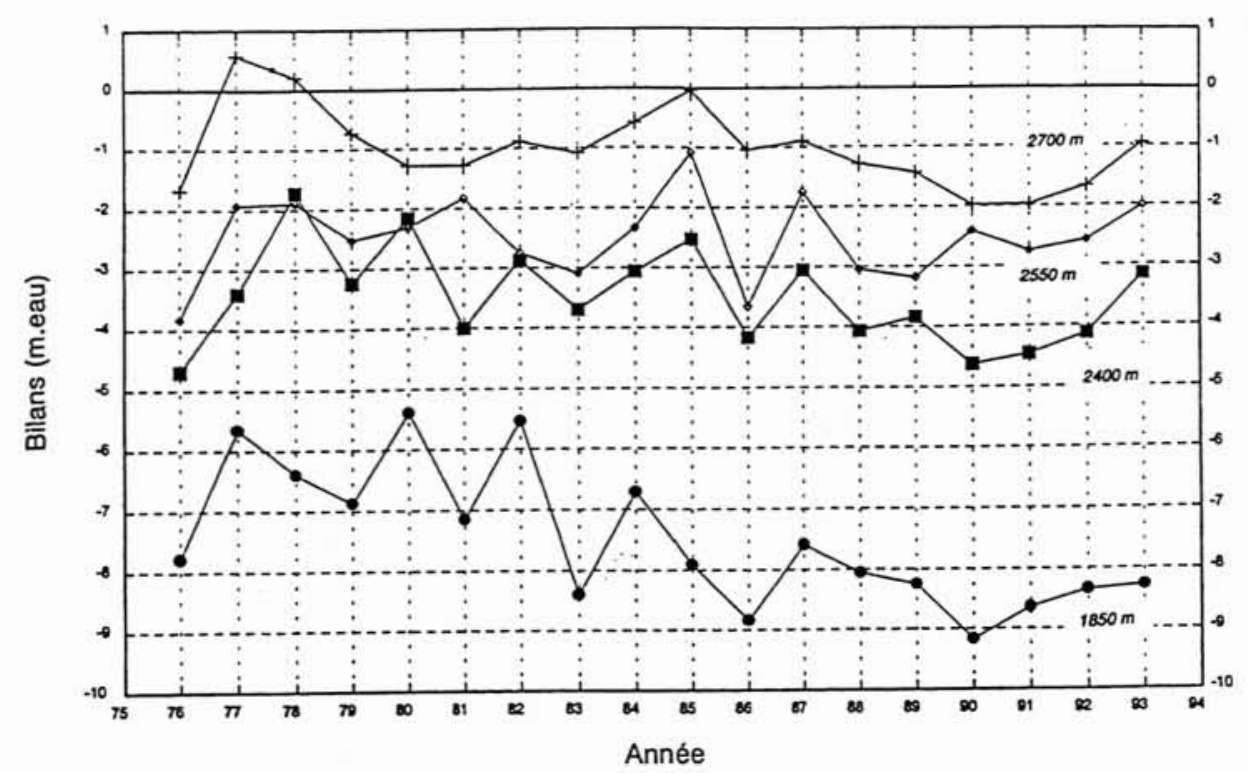

1. Evolution temporelle des bilans caractéristiques des quatre profils du glacier d'Argentière (1975-1993).

longitudinalement que transversalement) et les balises n'ayant pas été, chaque année, réimplantées rigoureusement aux mêmes points, la simple moyenne des bilans observés sur chaque profil est entachée d'un bruit considérable. Pour réduire l'incertitude sur les bilans caractéristiques de chaque région, nous avons appliqué, sur chaque profil, principes et méthodes utilisés par Lliboutry [11] pour l'analyse des bilans de masse sur le glacier de SaintSorlin (modèle linéaire des fluctuations spatiotemporelles). L'incertitude sur les bilans de masse moyens à chaque altitude peut être ainsi ramenée à environ 0,5 mètres d'eau par an ( $\sigma=0,3$ à $0,6 \mathrm{~m}$ suivant les profils et les époques).

Les principaux résultats de l'analyse statistique des 18 années d'observations sont les suivants :

1) Le modèle linéaire des variations de bilan de masse n'est pas vérifié, la variabilité interannuelle du bilan diminuant régulièrement avec l'altitude $(4 \sigma=5,08 \mathrm{~m}$ d'eau à $1850 \mathrm{~m}, 4 \sigma=3,76 \mathrm{~m}$ d'eau à $2375 \mathrm{~m}$, $4 \sigma=2,88 \mathrm{~m}$ d'eau à $2725 \mathrm{~m}$ ),

2) les fluctuations des bilans de masse n'en sont pas moins très fortement corrélées, $81 \%$ de la variance étant commune, d'origine a priori climatique, $12 \%$ seulement correspondant à une discrimination altitudinale (c'est cette très forte covariance qui peut faire penser que le modèle linéaire est une bonne approximation, compte tenu du «bruit» relativement important affectant les mesures de bilan),

3) les seules variables explicatives pertinentes sont:

a) les températures au cours de la saison d'ablation (mai à août) rendant compte, suivant les zones, de 30 à $53 \%$ de la variance,

b) à peine significatives au seuil de $5 \%$, les précipitations « hivernales " (octobre à mai) responsables de 8 à $12 \%$ de la variance.

Nous avons reporté sur la figure 2 les valeurs du coefficient de régression des bilans en les fluctuations de température. Les valeurs obtenues $\left(0,42\right.$ à $1,08 \mathrm{~m}$ d'eau. $\left.{ }^{\circ} \mathrm{C}^{-1}\right)$ sont comparables aux sensibilités observées par d'autres auteurs tant dans les Alpes qu'en Scandinavie. Compte tenu des incertitudes importantes dont sont entachées ces valeurs, il est difficile d'affirmer que la sensibilité diminue réellement avec l'altitude; néanmoins la présomption est très forte. A titre de comparaison, nous avons représenté deux courbes d'évolution de la sensibilité des bilans de masse aux variations de température. L'une est celle que l'on peut déduire de la simulation faite par Oerlemans [6] à partir de son modèle, l'autre dérive de la relation empirique entre ablation de glace et température moyenne pendant la saison d'ablation proposée par Krenke et Khodakov [13]. Le modèle d'Oerlemans surestime semble-t-il fortement la sensibilité des bilans de masse aux variations de température à basse altitude. Il s'agit probablement d'une conséquence de la sensibilité extrême de ce modèle aux variations de l'albedo du glacier et de la paramétrisation de l'albedo utilisée par l'auteur qui entraîne une augmentation très rapide du bilan énergétique lorsque l'on descend en dessous de la ligne d'équilibre du glacier. Par contre la relation empirique de Krenke et alt. rend compte de manière assez satisfaisante des observations faites tant sur le glacier d'Argentière, que $100 \mathrm{~km}$ plus au Sud, sur le glacier de Sarenne.

Bien que couvrant près de $900 \mathrm{~m}$ de dénivelé, notre réseau d'observation sur le glacier d'Argentière est encore trop peu étendu pour apprécier de manière réellement satisfaisante la sensibilité des bilans de masse aux fluctuations de températures. Pour ce faire, il serait nécessaire d'étendre les observations non seulement à la zone d'accumulation mais aussi aux régions de basse altitude, au voisinage immédiat du front des grands glaciers; la valeur de l'ablation au front étant un des paramètres importants régissant, selon Johannesson et al. [14], l'extension des glaciers et leur temps de réponse.

Les conséquences de l'incertitude sur la relation bilans de masse-température peuvent être appréciées sur la figure 3 où nous avons représenté, pour un grand glacier du massif du Mont Blanc, le glacier des Bossons, la variation de volume, cumulée du sommet du Mont Blanc au front du glacier, qui résulterait d'une variation de tem- 


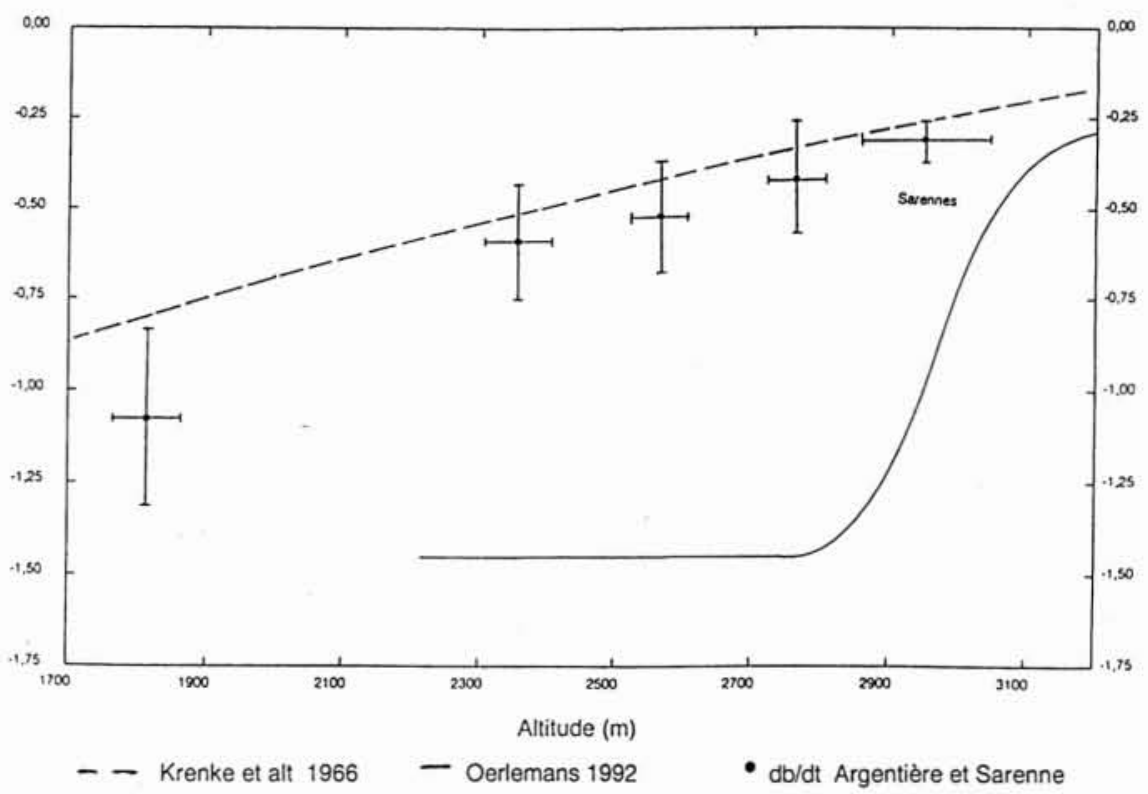

2. Relation bilan de masse-température observée sur le glacier d'Argentière en fonction de l'altitude.

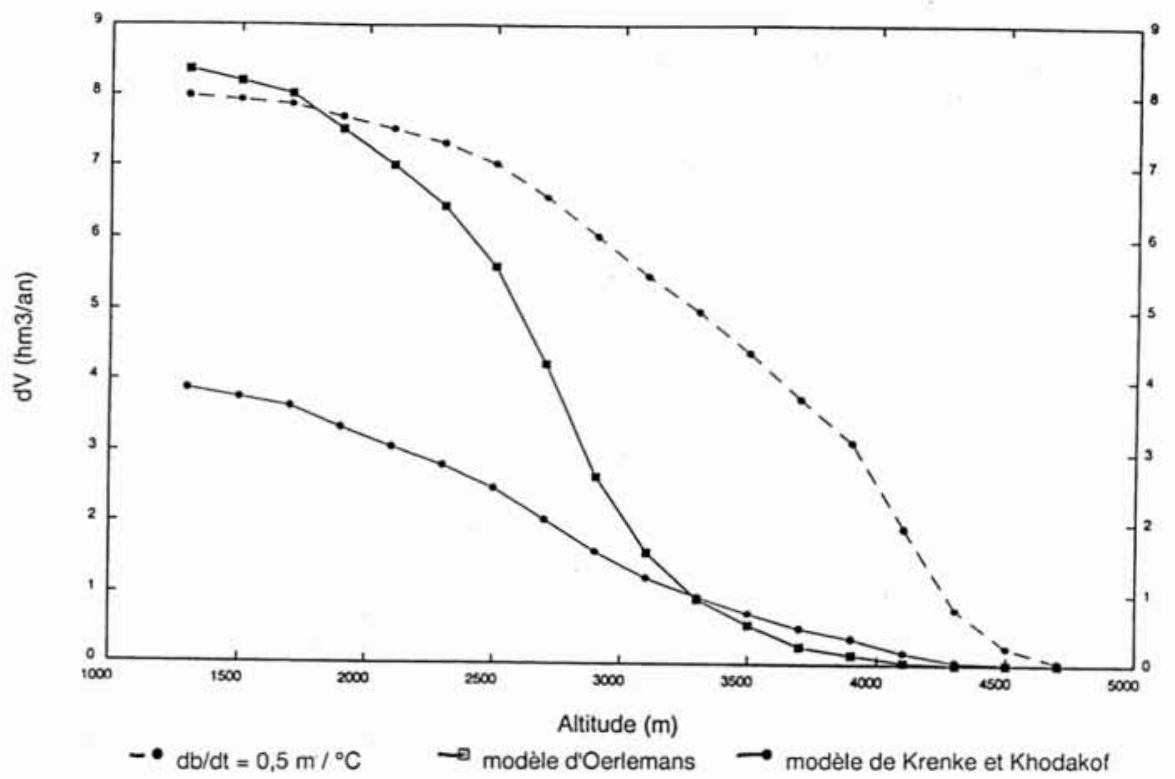

3. Variation de volume cumulée sur le glacier des Bossons pour une variation de température de $1^{\circ} \mathrm{C}$ et pour trois modèles différents.

pérature de $1^{\circ} \mathrm{C}$ selon trois hypothèses : modèle linéaire avec un coefficient de $0,5 \mathrm{~m}$ d'eau. ${ }^{\circ} \mathrm{C}^{-1}$, modèle de Oerlemans et relation de Krenke et Khodakov (proche de la relation observée sur le glacier d'Argentière). Le modèle linéaire et le modèle d'Oerlemans, bien que résultant de deux approches totalement différentes donnent des résultats remarquablement proches mais ils prévoient des variations de volume deux fois plus importantes qu'une relation compatible avec nos observations.

Quant à la sensibilité des bilans de masse glaciaires aux fluctuations des précipitations, elle paraît très faible, avec des pourcentages d'explication de la variance des bilans excessivement modestes $\left(\rho^{2} \simeq 0,08\right.$ à 0,12$)$ et des coefficients de régression non significativement différents de zéro au seuil de $5 \%$. Cette insensibilité apparente des bilans de masse est d'autant plus étonnante que la période étudiée est caractérisée par une variabilité importante des précipitations hivernales, avec les années les plus sèches et les plus arrosées du demi siècle. Mais ces quelques années extrêmes, concentrant l'essentiel de la variabilité climatique de la période sur deux variables bien corrélées (hivers humides et étés frais pour 1976-77 et 1977-78, hivers secs et étés chauds en 1975-76 et 1988-89), rendent difficile toute discrimination du rôle respectif de ces deux variables. Pour obtenir une relation fiable entre bilan de masse et précipitations, il nous paraît nécessaire d'estimer à l'avenir directement la relation accumulationprécipitations à différentes altitudes. 


\section{II $\square$ STABILITÉ DES RELATIONS BILANS DE MASSE-VARIABLES MÉTÉOROLO- GIQUES}

On peut remarquer que la corrélation entre bilans de masse et variables météorologiques est relativement modeste (coefficients de corrélation multiples compris entre 0,62 et 0,81 suivant les profils), alors que nombre d'études similaires affichent des coefficients plus confortables, de 0,85 à 0,95 . Le glacier d'Argentière et la sation météorologique de Chamonix constitueraient-ils un couple particulièrement mal choisi pour examiner la relation climat-bilans de masse glaciaire ? Serge Martin [15] ayant montré que le couple glacier de Sarenne-station météorologique de Lyon-Bron était assez bien accordé ( $77 \%$ d'explication de la variance sur la période 1949-1975) nous avons examiné l'ensemble des observations des bilans de masses du glacier de Sarenne de 1950 à 1991 publié par F. Valla [16]. En appliquant le même protocole que pour l'analyse des bilans de masse du glacier d'Argentière, on obtient les résultats rassemblés sur le tableau 1 .

On retrouve bien, pour la période 1950-1970, la relation bilan de masse-variables météorologiques publiée par $\mathrm{S}$. Martin, mais cette relation disparaît pendant la période suivante. S'il peut être réconfortant de trouver l'empreinte de la variabilité accrue des précipitations entre 1971 et 1991, il est par contre assez troublant d'assister à une
Tableau 1. - Part de variance des différents termes du bilan de masse du glacier de Sarenne "expliqués " par les fluctuations des variables météorologiques de la station de Lyon-Bron.

\begin{tabular}{|l|c|c|}
\hline & $\begin{array}{c}\text { Températures } \\
\text { estivales } \\
\text { (juillet-août) }\end{array}$ & $\begin{array}{c}\text { Précipitations } \\
\text { hivernales } \\
\text { (octobre à juin) }\end{array}$ \\
\hline $\begin{array}{l}\text { 1950-1970 } \\
\text { Bilans } \\
\text { Ablation } \\
\text { Accumulation }\end{array}$ & $\begin{array}{c}0,51 \\
\text { (c) }\end{array}$ & 0,13 \\
$\begin{array}{l}\text { 1971-1991 } \\
\text { Bilans } \\
\text { Ablation } \\
\text { Accumulation }\end{array}$ & 0,25 & 0,38 \\
\hline
\end{tabular}

diminution importante de la corrélation entre ablation de neige et de glace et température estivale alors que la variabilité des températures est comparable pour les deux périodes.

Une fenêtre glissante permet de situer l'époque de la chute du coefficient de corrélation vers 1973 (fig. 4). La médiocrité de la relation statistique entre bilans de masse et variables météorologiques pour le glacier d'Argentière entre 1976 et 1993 paraît moins originale.

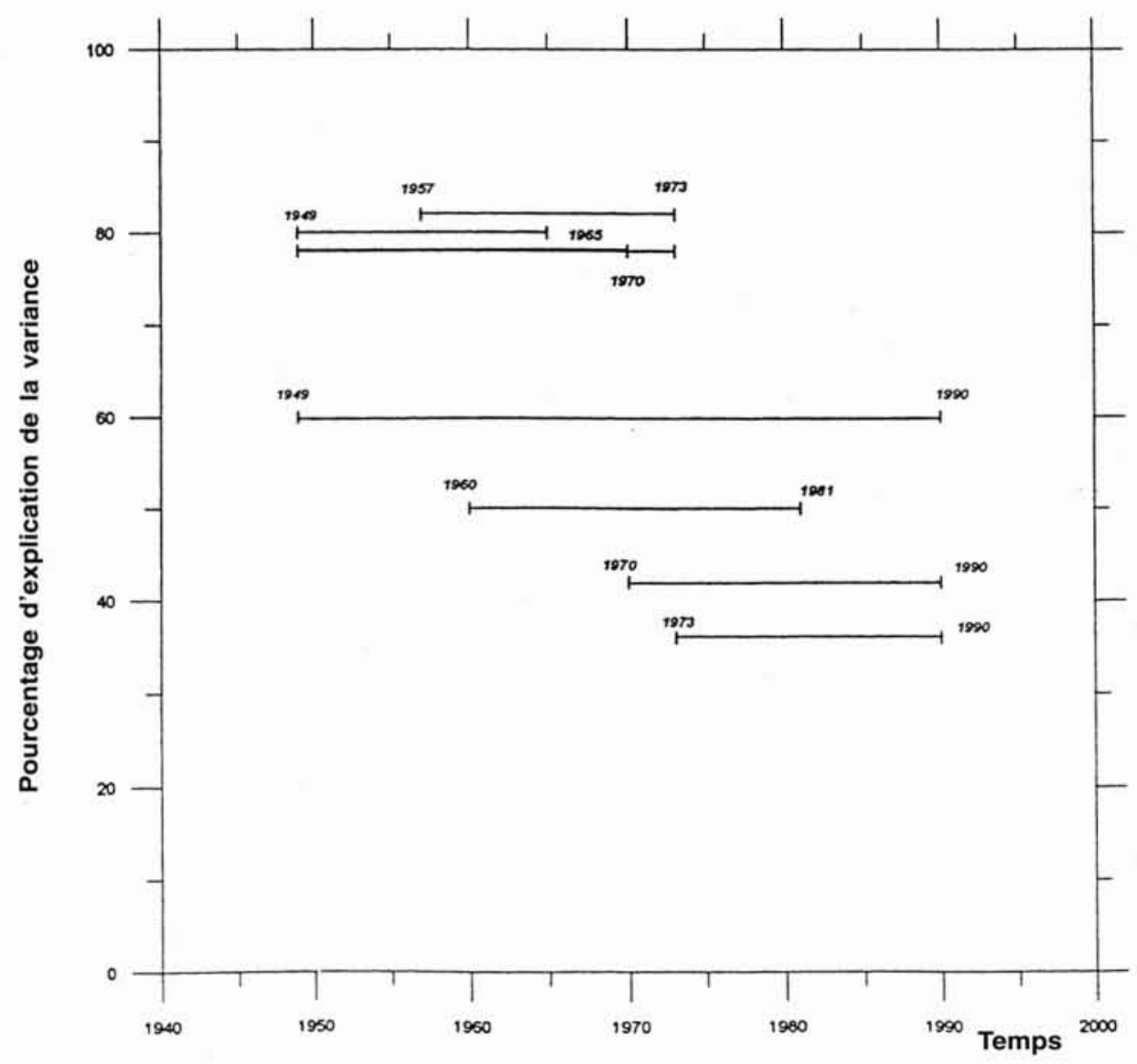

4. Pourcentage d'explication de la variance de l'ablation du glacier de Sarenne par la somme des températures estivales de Lyon-Bron supérieures à $17,5^{\circ} \mathrm{C}$ pour différentes périodes. 
En fait, la relation température estivale-ablation n'est pas une relation de causalité, compte tenu de la prédominance des échanges radiatifs à la surface du glacier en été. Cette relation ne peut être mise en évidence que s'il existe une très forte corrélation négative entre température et nébulosité. Cette relation s'est-elle estompée ou le climat au voisinage des stations météorologiques est-il moins représentatif de l'insolation en montagne depuis une vingtaine d'années?

\section{Remerciements}

L'étude des bilans de masse du glacier d'Argentière a été réalisée dans le cadre de contrats entre le Laboratoire de Glaciologie et Géophysique de l'Environnement du C.N.R.S. et Emosson S.A. qui a pris en charge le coût de toutes les opérations de terrain depuis 1975; la collaboration de M. Legland (C.G.C. au G.P.R.H.) ayant été particulièrement précieuse. Nous ne pouvons citer ici les dizaines de techniciens, chercheurs et étudiants qui ont participé aux différentes campagnes mais nous tenons à rendre hommage aux principaux acteurs de la première décennie d'observation (1976-85): C. Carle, D. Hantz, Y. Morin et R. Ribola.

\section{Références}

[1] WARrick R., Oerlemans J. (1990), - Sea level rise. Climate change, the IPCC scientific assessment, Houghton et all. Editors, Cambridge University Press, pp. 257-281.

[2] Trupin A., Meier M.F., Wahr J.M. (1992). - Effect of melting glaciers on the Earth's rotation and gravitational field: 1965-1984. Geophysical Journal International, 108, pp. 1-15.

[3] Oerlemans J., ForTUIN J.P.F. (1992). - Sensitivity of glaciers and small ice caps to greenhouse warning. Science, 258, pp. 115-117.

[4] MEIER M.F. (1993), - Ice, climate and sea level; do we know what is happening ? Ice in the climate system. Peltier Edt. Nato ASI, series 1, vol. 12.

[5] MARTIN S. (1974), - Corrélation bilans de masse annuelsfacteurs météorologiques dans les Grandes Rousses. Zeitschrift für Gletscherkunde und Glazialgeologie, Bd 10, pp. 89-100.
[6] Oerlemans J. (1992). - A model for the surface balance of ice masses : part I. Alpine glaciers. Zeitschrift für Gletscherkunde und Glazialgeologie. Bd 27/28, pp. 63-83.

[7] KUHN M. (1981). - Climate and glaciers. AIHS $\mathrm{n}^{\circ} 131$, pp. 3-20.

[8] Letreguilly A. (1984). - Bilans de masse des glaciers alpins: méthodes de mesure et répartition spatiotemporelle. Thèse de $3^{\mathrm{e}}$ cycle USTMG, $274 \mathrm{p}$. (inédit).

[9] CHEN J., FUNK M. (1990). - Mass balance of Rhonegletscher during 1982/83-1986/87. Journal of Glaciology, vol. $36, \mathrm{n}^{\circ} 123$, pp. 199-209.

[10] LLiBOUTRY L. (1974). - Multivariate statistical analysis of glacier annual balances. Journal of Glaciology 13, pp. 371392.

[11] KUHN M. (1984), - Mass budget inbalace as criterion for a climatic classification of glaciers. Geografiska Annaler. A 66, p. 229.

[12] GelBERT C. (1986). - Essai de reconstitution des bilans de masse glaciaires dans les Alpes depuis 1526. Mémoire de Diplôme d'Etudes Approfondies, Université Joseph Fourier, Grenoble. 90 p. (inédit).

[13] KRENKe A.M. et KhodAKov V.G. (1966), - Relation entre fusion superficielle des glaciers et température de l'air: Académie des Sciences de I'URSS, «Data on Glaciological Studies », vol. 12, pp. 153-164 (en russe).

[14] JOHANNESSON T., RAYMOND C., WADDINGTON E. (1989). Time scale for the adjustment of glaciers to changes in mass balance. Journal of Glaciology, vol. 35, $\mathrm{n}^{\circ} 121$, pp. 355-369.

[15] MARTINS S. (1977). - Analyse et reconstitution de la série des bilans annuels du Glacier de Sarennes, relation avec les fluctuations du niveau de 3 glaciers du Mont Blanc. Zeitschrift für Gletscherkunde und Glazialgeologie, Bd 13 , pp. 127-153.

[16] VAlLA F. (1994), - Sarennes. Un glacier sous haute surveillance. Revue de géographie alpine. Tome $81, \mathrm{n}^{\circ} 3$, pp. 33-49.

[17] MEIER M.F. (1984). - Contribution of small glaciers to global sea level. Science 226, pp. 1418-1421. 